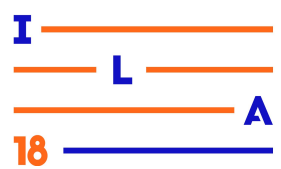

\title{
FLUXO OPERACIONAL DE TESTES DE USABILIDADE REMOTOS EM UM MARKETPLACE DE UX
}

\section{REMOTE USABILITY TESTING WORKFLOW FOR AN UX MARKETPLACE}

Mateus Filipe de Lima Pelanda ${ }^{1}$, Bach. Sávio Correia ${ }^{2}$, Bach.

Rafael Zabotini Venjenski ${ }^{3}$, Bach.

Frederick Marinus Constant Van $\mathrm{Amstel}^{4}$, D.Sc.

(1) Pontifícia Universidade Católica do Paraná - PUCPR e-mail: mateuspelanda@gmail.com

(2) Pontifícia Universidade Católica do Paraná - PUCPR e-mail: saviofc@gmail.com

(3) Pontifícia Universidade Católica do Paraná - PUCPR e-mail: rafaelzabotini@gmail.com

(4) Pontifícia Universidade Católica do Paraná - PUCPR e-mail: frederick.amstel@pucpr.br

Palavras-chave em português (teste remoto, usabilidade, marketplace)

A demanda por especialistas em experiência do usuário para o aperfeiçoamento de aplicativos mobile é crescente no Brasil, tornando-se necessária a criação de novas ferramentas e métodos para essa atividade profissional. Este artigo relata o desenvolvimento de um fluxo operacional para testes de usabilidade remotos não-moderados dentro de uma plataforma profissional do tipo marketplace.

\section{Key-words in English (remote test, usability, marketplace)}

The demand for user experience experts, for the improvement of mobile applications, is growing in Brazil, making it necessary to create new tools and methods for them. This article reports the development and operational flow for a non-moderated remote usability testing within a marketplace platform.

\section{Introdução}

Usabilidade pode ser definida como a qualidade de uso de algum produto do ponto de vista da experiência do usuário (KRUG, 2008). Para a avaliação desta qualidade, especialistas em experiência do usuário costumam utilizar como método o teste de usabilidade, que consiste em apresentar o produto para um grupo de pessoas e solicitar que os mesmos tentem executar tarefas. Os testes podem ser realizados na modalidade presencial, com a presença física do usuário em um ambiente de laboratório ou em ambiente de trabalho do mesmo. Testes também podem ser realizados na modalidade remota, com ou sem a moderação de um especialista (CARDOSO, 2011).

Embora o teste de usabilidade seja altamente recomendado pela literatura, este não é o único método utilizado para avaliar e definir qualidades relativas à experiência do usuário. Uma pesquisa realizada com especialistas brasileiros, revelou que no Brasil, os métodos mais utilizados são: entrevistas em profundidade, jornada do consumidor, testes de usabilidade presencial, design sprint e testes de usabilidade remotos (LESLIE, 2018). Embora sejam bastante conhecidos e disseminados, nem sempre os especialistas conseguem executar estes métodos em seus projetos. 


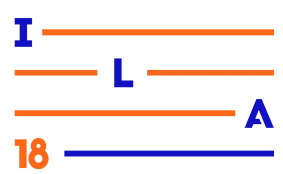

Dentre os maiores desafios, são apontados a aceitação da incerteza proveniente dos processos iterativos, a criação de uma cultura de experiência do usuário na empresa e a falta de mão de obra qualificada (LESLIE, 2018).

Apesar dessas dificuldades, a demanda por especialistas em experiência do usuário é crescente. Tais especialistas ainda são confundidos com designers gráficos especializados em interfaces e pouco valorizados pela sua capacidade de trabalhar com aspectos intangíveis do projeto de um produto (BATTISTELLI, 2018). O conhecimento sobre a atividade desse tipo de profissional ainda não foi o suficiente para a criação de ferramentas adequadas para o exercício da profissão. Existem várias ferramentas, porém, elas não se integram e não ajudam o profissional a colaborar com profissionais de outras áreas. O teste de usabilidade, em particular, é uma atividade do especialista em experiência do usuário que poderia ser melhor suportada por ferramentas integradas.

Nos próximos tópicos serão exploradas as relações entre a demanda do mercado por especialistas e a desintegração das ferramentas, que resulta em custos elevados para a realização da atividade de testes de usabilidade. A proposta dessa pesquisa é definir um fluxo operacional para esse tipo de teste, visando baratear seu custo e ampliar o acesso ao método através de uma plataforma online que está sendo desenvolvida pelos autores.

\section{O problema da falta de recursos humanos}

Ao longo dos últimos 20 anos, o campo de experiência do usuário evoluiu em todo o mundo, porém, no Brasil, essa evolução foi tardia. Há uma intensa demanda nos últimos 5 anos, porém, sem investimentos ordenados em formação especializada e sem a valorização de profissionais experientes (TEIXEIRA, 2016). A demanda por profissionais na área triplicou no primeiro semestre de 2017

(TEIXEIRA, 2017). Esse profissional é fundamental para fazer a ponte entre as equipes responsáveis pelo projeto e pela produção de produtos digitais, sendo eventualmente alocado como parte da equipe de projeto ou como uma equipe separada.

A pesquisa Panorama do Mercado de UX no Brasil, realizada pela consultoria especializada em experiência do usuário Saiba + , revelou que as grandes empresas detém hoje a maioria dos especialistas em experiência do usuário disponíveis no mercado (37\%), estando o restante diluído entre startups, pessoas jurídicas, consultorias e freelancers (LESLIE, 2018). Além disso, 57\% da mão de obra especializada está localizada em São Paulo, seguido por Santa Catarina com $11 \%$ e o Rio de Janeiro com 9\% (Figura 01).

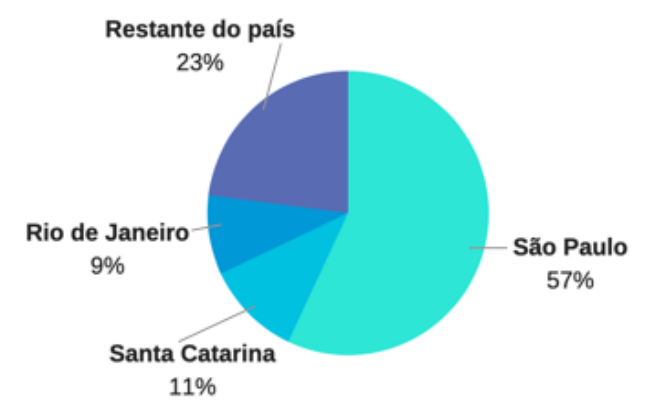

Figura 01 - Quantidade de especialistas em experiencia do usuário espalhados pelo território brasileiro.

Fonte: Adaptado de Panorama do Mercado de UX no Brasil (2018)

Dos profissionais que responderam a pesquisa, cerca de $32 \%$ se consideram sênior, enquanto $42 \%$ se consideram júnior ou pleno. $44 \%$ estão a menos de 3 anos exercendo essa profissão, $43 \%$ entre 4 a 10 anos e $12 \%$ os que estão a mais tempo, apresentados na Figura 02.

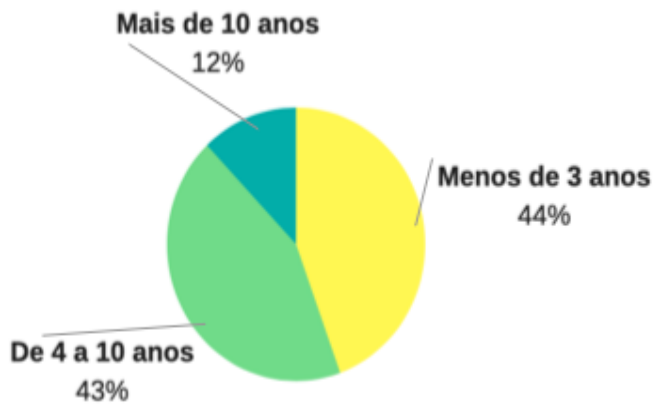

Figura 02 - Quantidade de profissionais pelo tempo de profissão.

Fonte: Adaptado de Panorama do Mercado de UX no Brasil (2018) 


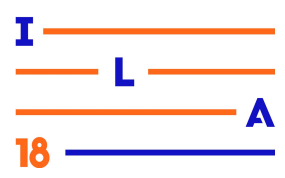

A análise dos dados levantou a hipótese de que especialistas em experiência do usuário possam estar sendo pressionados a evoluir na carreira muito rapidamente, se tornando sênior com menos de 3 anos de carreira. Outra hipótese é que o mercado não está sabendo diferenciar de um profissional experiente de um novato, o que pode comprometer a qualidade do trabalho profissional (LESLIE, 2018). O teste de usabilidade, em particular, é uma atividade fundamental que costuma ser negligenciada ou enviesada, diminuindo a credibilidade da profissão.

Grandes empresas costumam atender seus clientes em todo o território brasileiro a partir de São Paulo. Porém como há menos especialistas em outros Estados, o teste de usabilidade remoto tem sido uma maneira de mitigar a distribuição desigual de recursos humanos especializados em experiência do usuário no Brasil. A variante não-moderada, que será explicada a seguir, ainda é uma novidade para o mercado brasileiro que tende a crescer neste cenário.

\section{Teste de usabilidade remoto não-moderados}

Testes de usabilidade podem ser executados a distância, sendo assim denominados de testes remotos, dispensando recursos físicos, tais como laboratórios e os custos de deslocamento necessários para a presença do usuário. Estes testes podem ser moderados ou não moderados.

No teste remoto moderado, o usuário conversa com $\mathrm{o}$ aplicador do teste enquanto executa as tarefas, enquanto que no teste não-moderado, as instruções para a realização das tarefas são apresentadas na tela do usuário diretamente, como parte do produto ou como um acessório do mesmo (HARTSON, 1996). Owen (2016) aponta como sendo grande o número de fatores levados em consideração na hora de definir que tipo de teste será aplicado. Testes remotos não-moderados apresentam algumas vantagens em relação aos testes tradicionais realizados em laboratório:
- Diversidade geográfica de participantes;

- Custos reduzidos;

- Entrega e aplicação de testes de maneira ágil; e

- Possibilidade de testes dependentes de contextos.

As desvantagens segundo Schade (2013) são:

- O teste fica sujeito a interrupções de rotina, comprometendo os resultados;

- O desempenho de processamento do aparelho do usuário pode interferir nos resultados;

- Falta de suporte em tempo real;

- O usuário pode se sentir pouco à vontade com a ferramenta de teste; $\mathrm{e}$

- Diminuição do envolvimento da equipe com o realizador do teste.

Outros estudos demonstram problemas semelhantes encontrados nos testes tradicionais de laboratório. Dentre estes, é possível relacionar tais problemas a contaminação do público selecionado, ou então aos testes serem condicionados em um ambiente controlado, no qual os usuários se sentem na obrigação de contribuir da melhor maneira possível, sem questionar ou demonstrar dificuldades (CHYNAL e SOBECKI, 2015).

Atualmente, para tornar possível a execução de testes de usabilidade remotos, é necessário utilizar várias ferramentas no mercado, tanto para produtos quanto para serviços digitais (CARQUEIJA, 2016). Sendo assim, torna-se indispensável um estudo das ferramentas disponíveis no mercado e como seria um fluxo ideal que atendesse todas as demandas necessárias para a execução de testes e do aperfeiçoamento de produtos, seguindo uma metodologia a qual atende e compreenda o mercado em si. Utilizaremos aqui o método de benchmarking competitivo para medir e comparar produtos, serviços e processos de trabalhos de empresas (MADEIRA, 1999). 


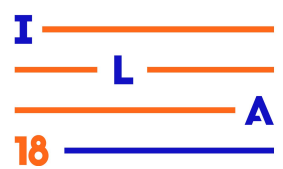

Para a realização desse estudo de benchmarking, foram recolhidas informações sobre algumas ferramentas existentes no mercado que realizam etapas do processo de teste de usabilidade - tais como prototipagem, recrutamento, análise de dados - indicadas por especialistas em experiência do usuário em blogs. As ferramentas analisadas foram:
FullStory, CanvasFlip, Testr, Morae, Usertesting, UXTesting, SmartLook, Lookback, Koncept.

Nesta avaliação, foram analisadas e mapeadas quais as plataformas de atuação, o tipo de serviço proposto pela empresa, integração com outras ferramentas, as fases suportadas do fluxo e os tipos de dados coletados (Tabela 1)

\begin{tabular}{|c|c|c|c|c|c|}
\hline & Plataforma & Serviço & Integração & $\begin{array}{c}\text { Etapas } \\
\text { suportadas }\end{array}$ & $\begin{array}{l}\text { Dados } \\
\text { Coletados }\end{array}$ \\
\hline Full Story & Web & $\begin{array}{l}\text { Monitoramento e } \\
\text { relatório de uso }\end{array}$ & $\begin{array}{c}\text { Trello, Slack, } \\
\text { Google Analytics e } \\
\text { outros }\end{array}$ & $\begin{array}{l}\text { Análise de dados } \\
\text { quantitativos }\end{array}$ & Vídeo de teste \\
\hline Canvasflip & $\begin{array}{l}\text { Web } \\
\text { Plugin } \\
\text { Mobile }\end{array}$ & $\begin{array}{l}\text { Prototipagem e testes } \\
\text { de usabilidade não } \\
\text { moderados }\end{array}$ & $\begin{array}{l}\text { Sketch, Balsamiq e } \\
\text { Slack }\end{array}$ & $\begin{array}{c}\text { Teste e análise de } \\
\text { dados } \\
\text { quantitativos }\end{array}$ & Mapa de calor \\
\hline Testr & $\begin{array}{l}\text { Web } \\
\text { Mobile }\end{array}$ & $\begin{array}{l}\text { Teste de qualidade de } \\
\text { software remoto e não } \\
\text { moderado, teste de } \\
\text { usabilidade remoto e } \\
\text { não moderado }\end{array}$ & - & $\begin{array}{c}\text { Recrutamento, } \\
\text { Teste e análise de } \\
\text { dados quantitativos }\end{array}$ & $\begin{array}{l}\text { Vídeo do teste } \\
\text { e mapa de } \\
\text { calor }\end{array}$ \\
\hline Usertesting & Web & $\begin{array}{l}\text { Teste de usabilidade } \\
\text { remoto }\end{array}$ & $\begin{array}{l}\text { inVision e uploads } \\
\text { nos formatos .ipa e } \\
\text {.apk }\end{array}$ & $\begin{array}{c}\text { Recrutamento, } \\
\text { Teste e análise de } \\
\text { dados quantitativos }\end{array}$ & Vídeo do teste \\
\hline UXtesting & $\begin{array}{l}\text { Web } \\
\text { Mobile }\end{array}$ & $\begin{array}{l}\text { Teste de usabilidade } \\
\text { remoto não-moderado }\end{array}$ & - & $\begin{array}{c}\text { Recrutamento, } \\
\text { Teste e análise de } \\
\text { dados quantitativos }\end{array}$ & Vídeo do teste \\
\hline Smartlook & $\begin{array}{l}\text { Web } \\
\text { Mobile }\end{array}$ & Teste de usabilidade & $\begin{array}{l}\text { Intercom, Mixpanel, } \\
\text { Zendesk, Google } \\
\text { Analytics, Google } \\
\text { Tag Manager, } \\
\text { Magento, Prestasho, } \\
\text { Shopify e Wordpress }\end{array}$ & $\begin{array}{l}\text { Análise de dados } \\
\text { quantitativos }\end{array}$ & Vídeo do teste \\
\hline Lookback & $\begin{array}{l}\text { Desktop } \\
\text { Mobile } \\
\text { Web }\end{array}$ & $\begin{array}{l}\text { Teste de usabilidade } \\
\text { remoto e não moderado }\end{array}$ & - & $\begin{array}{l}\text { Análise de dados } \\
\text { quantitativos }\end{array}$ & Vídeo do teste \\
\hline Koncept & $\begin{array}{l}\text { Web } \\
\text { Mobile }\end{array}$ & $\begin{array}{l}\text { Prototipagem e teste de } \\
\text { usabilidade remoto }\end{array}$ & - & $\begin{array}{l}\text { Teste e análise de } \\
\text { dado quantitativos }\end{array}$ & $\begin{array}{l}\text { Vídeo do teste } \\
\text { e mapa de calor }\end{array}$ \\
\hline
\end{tabular}

Tabela 01 - Benchmark de ferramentas no mercado. 


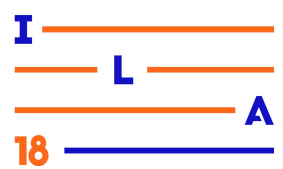

\section{Fluxo operacional para testes de} usabilidade remotos não-moderados

A partir dos dados coletados na pesquisa de benchmarking e de revisão sistemática de artigos científicos descrita por Kitchenham (2004) e publicações de sites especializados no tema, como Nielsen Norman Group, ACM Digital Library, ScienceDirect, Springer e artigos publicados no portal da CAPES, foi possível identificar as principais necessidades dos especialistas em experiência do usuário para o fluxo operacional de testes de usabilidade remotos não-moderados: a integração entre recrutamento, realização dos testes e a análise de resultados.

Para a elaboração deste fluxo operacional para testes de usabilidade não-moderados, foi utilizada a ferramenta de fluxograma de interação.

Fluxogramas de interação demonstram as etapas de execução de uma determinada tarefa, podendo ser utilizados para definir e avaliar as sequências de interações (BROWN, 2010). O principal objetivo de tal ferramenta, é o de ajudar na visualização do sistema como um todo, fazendo-se quando necessário a divisão de um fluxograma para cada tarefa e a utilização das primeiras etapas no topo superior esquerdo, descendo até o canto inferior direito (JORGE e MIYAKE, 2014).

A Figura 1 apresenta um fluxograma de como os testes remotos não-moderados podem ser realizados. Por responsável compreende-se a pessoa que cria a demanda do teste e por testador uma pessoa com perfil selecionado para determinado teste. $\mathrm{O}$ especialista é um profissional da área de experiência do usuário, enquanto a plataforma é a organização que adota o fluxo operacional. Este esquema é formado por 4 etapas principais, sendo estas a preparação, o recrutamento, o teste e a análise de resultados. Dentre estas, estão contemplados todos os passos desde a submissão do protótipo até o envio do relatório final, assim caracterizando o fluxo como um todo.

Nas seções a seguir serão descritas em maior detalhe cada uma das etapas principais deste fluxo.

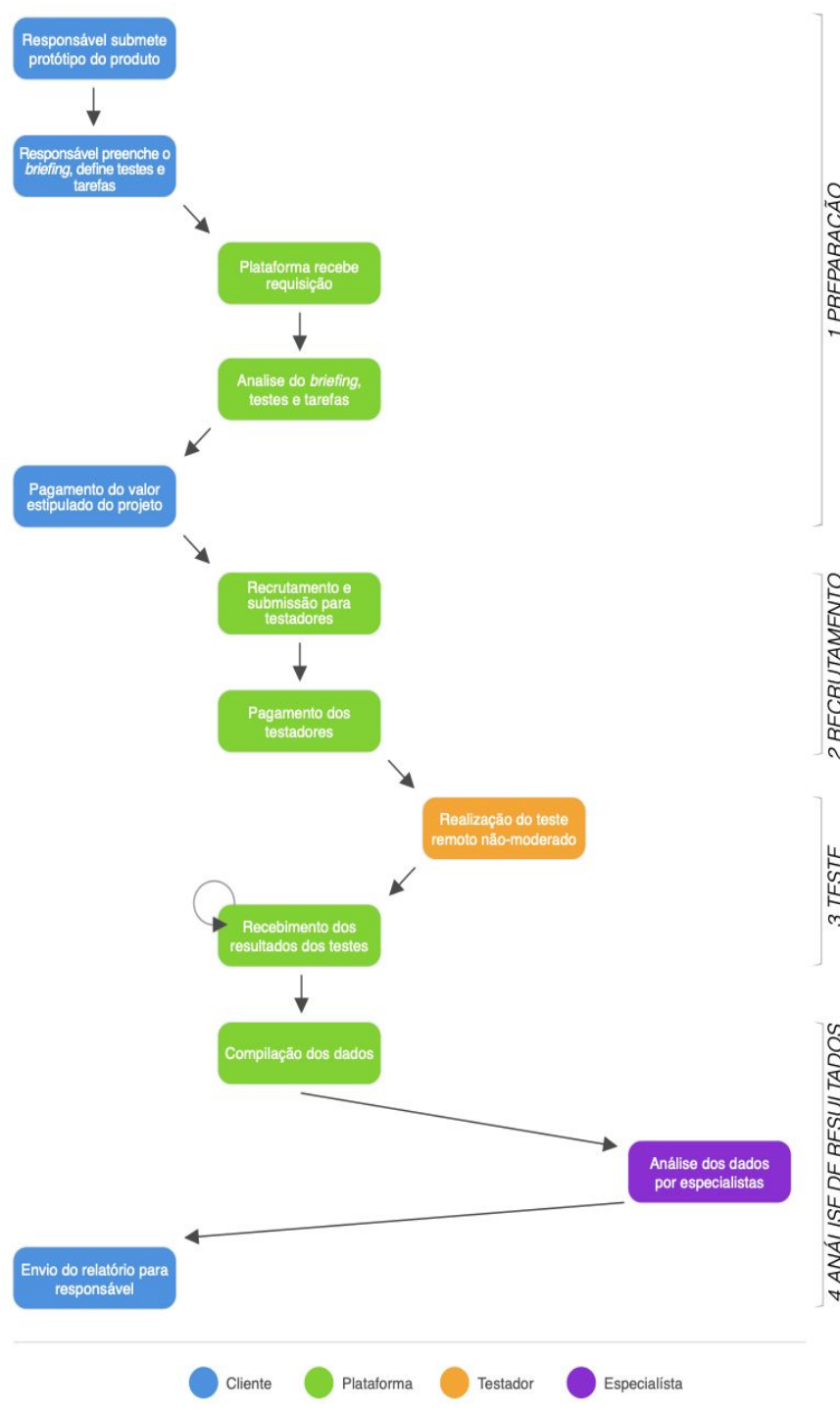

Figura 1 - Fluxograma de testes de usabilidade remotos não-moderados

\subsection{Preparação}

O primeiro contato se dá entre o responsável pela submissão de algum produto digital que deseja testar um protótipo, o cliente. $\mathrm{O}$ cliente precisa preencher um briefing com campos pré-definidos que permitam a contextualização do produto. Além disso, deve haver no planejamento do teste a definição das tarefas que o usuário testador irá executar e as características detalhadas do perfil do usuário. $\mathrm{O}$ cliente também precisa enviar o protótipo do produto nos formatos suportados pela plataforma. Com base nessas informações, o teste pode ser 


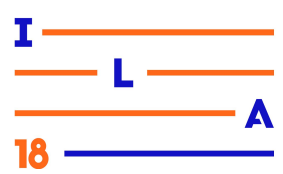

agendado, os testadores podem ser recrutados e os especialistas convidados.

\subsection{Recrutamento}

O ponto de partida para o recrutamento são definidos no briefing do teste, ou seja, quais tarefas serão realizadas, o nível de conhecimento desejado sobre o produto ou o nível de especialidade em usabilidade.

Os testes de usabilidade geralmente passam pelos testadores, potenciais usuários do produto final, sempre focando na possibilidade de encontrar falhas a serem reparadas. Atualmente é comum que o recrutamento ocorra com base em comportamentos e referências culturais dos testadores, além dos dados demográficos. Por exemplo, o tempo gasto em smartphones, o tipo de atividade que realiza online, como pedir comida ou solicitar serviços de transporte, ou gerir relacionamentos pessoais através de aplicativos e entre outros comportamentos.

\subsection{Teste}

O teste poderá ser realizado de maneira remota no ambiente natural do testador e deverá ser realizado na hora mais oportuna para o mesmo, sendo limitado à utilização dos equipamentos, como smartphones ou computadores. Quando remotos, devem ter curta duração, pois o testador pode perder o interesse em realizá-lo, visto que o teste é realizado sem moderador. O número de testadores pode variar de acordo com os objetivos definidos por quem submeteu o protótipo.

\subsection{Análise de Resultados}

A entrega do relatório de análise dos resultados são baseadas em atributos (e.g., eficiência, eficácia, erros, simplicidade, satisfação, etc.) sendo definidos na concepção dos testes e dos objetivos desejados, podendo variar de acordo com as necessidades do responsável que envia o protótipo.

Os dados gerados a partir dos testes podem ser tabulados de maneira automatizada, ao que se refere aos dados quantitativos (tais como o tempo para a conclusão de uma tarefa, taxa de sucesso e número de erros). Os dados qualitativos serão gerados pela análise dos especialistas convidados para atuar no projeto. Tanto os dados quantitativos, quanto os qualitativos serão utilizados para a análise de resultado dos testes, visando a identificação de problemas de usabilidade e demonstrando os principais defeitos, para assim viabilizar as soluções possíveis.

\section{Marketplace de UX}

A partir do fluxo operacional idealizado, é possível resolver a dificuldade da integração entre as ferramentas, mas ainda assim, permanece o problema da falta de recursos humanos. Como solução, o fluxo pode ser implementado em uma plataforma online baseada no modelo de negócio marketplace.

Marketplace é um ambiente de varejo online onde são ofertados produtos e serviços por diversos atores. A plataforma conecta e executa a transação entre compradores e vendedores e, por isso, cobra uma pequena taxa de operação (GIBERTONI 2014; VILHA, 2002). O conceito de marketplace foi criado pela empresa eBay, em 1995, surgindo inicialmente da modernização de leilões, sendo seguido pela Amazon, a qual formalizou o conceito, tornando os concorrentes da loja virtual em parceiros de venda (HUNGRIA, 2014).

Um marketplace voltado ao mercado de experiência do usuário pode ajudar a ampliar o acesso e visibilidade dos serviços profissionais da área. A plataforma de UX seguiria uma linha similar a das plataformas de transporte pessoal, como Uber e Cabify. Os profissionais contratados sob demanda seriam testadores e especialistas.

O diagrama apresentado na Figura 2, demonstra como funcionaria um modelo marketplace de plataforma online para testes de usabilidade remotos não-moderados. Os clientes enviam seus protótipos 


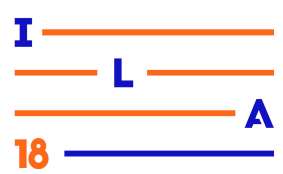

para a plataforma, estabelecem as tarefas que desejam ser testadas e realizam o pagamento de acordo com os serviços necessitados. Nesse processo, o cliente estabelece também o perfil do testador desejado. A plataforma vai, então, em busca de especialistas adequados e disponíveis para analisar o teste. Além da plataforma realizar as transações financeiras, ela funcionaria como orientadora no processo de testes, oferecendo suporte para ambos os lados, tanto cliente quanto testador.

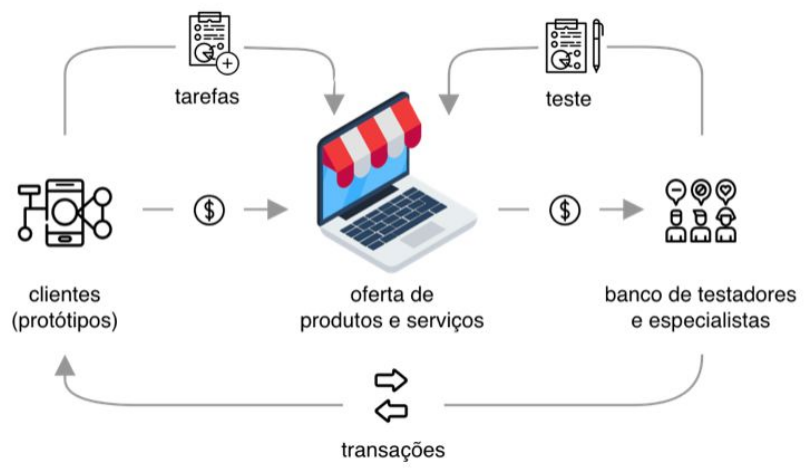

Figura 2 - Fluxo de interações em uma plataforma do tipo marketplace voltada para a realização de testes de usabilidade remotos não-moderados.

Com essa plataforma, especialistas em experiência do usuário podem atender clientes em qualquer região, utilizando o fluxo operacional otimizado para o trabalho à distância. Isso permitirá que profissionais que não estão localizados em grandes centros possam iniciar sua carreira na área ou se aperfeiçoar sem precisar se mudar para grandes centros. Por outro lado, existe a possibilidade dessa ferramenta gerar certa dependência e estagnação profissional caso não haja no futuro outros métodos de trabalho disponíveis na plataforma. Outra dificuldade importante é a seleção destes profissionais e a avaliação da qualidade de seu trabalho, que pode variar muito.

\section{Discussão}

Tradicionalmente, os testes de usabilidade ainda são realizados na modalidade presencial e costumam ser orientados por um facilitador. Para projetos com públicos dispersos geograficamente, ou que possuam a necessidade de obter resultados rápidos, com dados quantitativos, torna-se mais atraente a modalidade remota. Apesar disso, o problema da falta de integração entre as ferramentas ainda torna o teste de usabilidade remoto pouco viável.

Para que as ferramentas de teste de usabilidade remotos sejam integradas, é preciso implementar o operacional aqui proposto. Além disso, é preciso integrar estas ferramentas a uma plataforma digital que aja como facilitadora entre especialistas e testadores. Os profissionais de experiência do usuário que hoje são forçados a migrarem para grandes centros urbanos, por conta de não possuírem grandes oportunidades de trabalhos em suas cidades, adquirem a vantagem de não necessitarem de emigração para progredir na carreira.

Entende-se que há uma rotina para testes de usabilidade, sejam eles moderados ou não. A existência de uma plataforma que embarcasse a rotina dos testes de usabilidade e que também formasse um modelo de negócio marketplace, poderia fomentar uma comunidade de práticas e conhecimentos.

Contudo, essa junção também traz algumas questões polêmicas para os especialistas em experiência do usuário, principalmente sobre como se posicionar e ser reconhecido no mercado.

\subsection{Testes de usabilidade não-moderados oferecem resultados confiáveis para a tomada de decisão sobre $U X$ ?}

Atualmente, testes não-moderados são vistos ainda como pouco conclusivos para problemas complexos, pois estes são testes rápidos, não são aprofundados e seguem um roteiro pré-determinado (qual não pode ser modificado durante o andamento do teste). Mas mesmo assim, eles são bem vistos para problemas pontuais, como é o caso de itens de interfaces em desenvolvimento ou para fluxo de navegação. Supondo que inicialmente uma solução deste tipo 


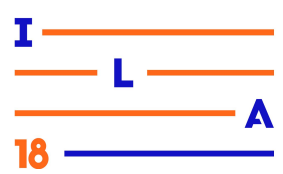

atingiria principalmente produtos em fase inicial de prototipagem, existe um grande potencial para que o desenvolvimento de aplicativos passem por testes remotos, como no caso do fluxo desenhado anteriormente, para a entrega de um produto digital com menos falhas e maiores chances de retenção de usuários. As ferramentas disponíveis no mercado, que foram analisadas nessa pesquisa, proporcionam dados, como complexidade de menus ou dificuldades de navegação, capazes de embasar tomadas de decisão com argumentos práticos.

\subsection{Que qualidades observadas em teste de usabilidade podem ser convertidas em métricas automatizadas?}

Quando trata-se de testes remotos não-moderados, pensa-se em atingir um número elevado de testadores para perceber falhas repetitivas, dificuldades de navegação e o tempo de interação. Logo, os dados quantitativos, podem ser analisados por meio de algoritmos a serem alimentados pelos inputs da plataforma, tratados e aplicados no produto (fundamentados em teorias de testes já existentes e então trazidas às plataformas digitais como relatórios automatizados). Portanto, o cruzamento destes dados, trariam estatísticas mais tangíveis para o campo da usabilidade.

\section{3 É possível "Uberizar" o trabalho de profissionais de $\mathrm{UX}$ ?}

Ao pensar no mercado de trabalho, há um consenso geral de que especialistas em experiência do usuário estão concentrados, em sua maior parte, em grandes centros como São Paulo. A proposta de uma plataforma que disponibiliza especialistas remotamente traz possibilidades de alavancar os testes de usabilidade em todo o país, quiçá no mundo.

Uma das principais vantagens do teste remoto é ser mais acessível, tanto do ponto de vista financeiro, quanto geográfico, sendo que estas características podem ter uma conotação negativa, pois pode parecer uma banalização dos testes. Em contrapartida, a entrada de profissionais da área de experiência do usuário que já atuam no mercado agregam credibilidade e segurança de que os testes serão realizados por pessoas credenciadas provindas de um nicho tradicional de mercado já consolidado.

O desenvolvimento de uma comunidade em um espaço de marketplace, onde os próprios especialistas da área seriam desta base, não os tornam concorrentes, pois existiriam assim, diferentes níveis de especialidades desejadas, assim como aquelas procuradas em diferentes partes do processo de validação. Dentro deste ambiente, é possível criar um espaço de aprendizado, assim como originar potenciais usuários de algum determinado aplicativo, que esteja em fase de teste e que desejam testar seu produto digital com esses tipos de usuários leigos. A criação de uma comunidade de especialistas pode gerar mais oportunidades de difundir os testes de usabilidade em escalas maiores. Também, continua-se indispensável a opinião de profissionais da área de experiência de usuário dentro de empresas, pois produtos necessitam de outras formas de validações e cuidados em relação às interações.

\subsection{Inteligências artificiais poderiam tomar o lugar de profissionais de UX?}

A obtenção de dados a partir do marketplace, podem trazer vantagens a todo o mercado de experiência de usuários, possibilitando a automatização de relatórios de testes e na elaboração de projetos. As inteligências artificiais já são uma realidade no contexto de automatização de tarefas com ferramentas que facilitam a criação e até mesmo na análise de produtos. A elaboração de layouts e desenvolvimento de sistemas possuem métricas semelhantes, convergindo o design para um mesmo ponto em comum, tendendo a obedecer os mesmos padrões de estruturas de informação. $O$ desenvolvimento de plataformas BIM (Building Information Modeling), que consistem em blocos de informações, formas e estados de objetos (utilizado 


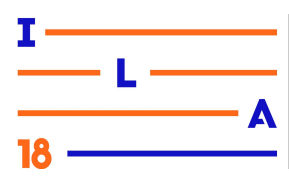

em software de Construção Civil), é um exemplo da geração de design automatizado que torna obsoleto parte do trabalho de engenheiros e arquitetos.

O machine learning (aprendizado de máquinas), que segue tendências e padrões, a partir de dados coletados de usuários, e a utilização de comandos de voz para execução de tarefas, tornam cada vez mais próximos um futuro onde a elaboração de projetos se torna totalmente autônoma, seguindo as especificidades de clientes e obedecendo a padrões de usabilidade pré-estabelecidos. Sendo assim, o profissional que somente elabora e avalia produtos pode se tornar obsoleto. Por outro lado, este profissional pode também abraçar as oportunidades de desenvolvimento de ferramentas automatizadas de design. Acreditamos que, em um futuro próximo, especialistas em experiência de usuário se tornarão especialistas em metadesign, ou seja, capazes de projetar sistemas de alta complexidade e alta abstração, tal como vislumbrado por Caio Vassão em seu livro "Metadesign: ferramentas, estratégias e ética para a complexidade" (VASSÃO, 2010).

\section{Referências Bibliográficas}

BATTISTELLI, Juliana. 5 motivos mais do que justos para se tornar um UX designer. Disponível em:

$<$ https://blog.mastertech.tech/design/5-motivos-mais -do-que-justos-para-se-tornar-um-ux-designer/> Acessado em 16 de julho de 2018.

\section{BATTISTELLI, Juliana. Com um UX designer sua} empresa terá melhores resultados (não só mais bonitos!). Disponível em

$<$ https://blog.mastertech.tech/negocios/com-um-uxdesigner-sua-empresa-tera-melhores-resultados-naoso-mais-bonitos/> Acessado em 16 de julho de 2018 .

BOGAN, Christopher E. e ENGLISH, Michael J. 1994. Benchmarking for Best Practices: Winning Through Innovative Adaptation (1nd. Rd.). McGraw Hill, New York, USA,
(ISBN-10),0070063753.

BROWN, Dan M. Communicating design: developing web site documentation for design and planning. New Riders, 2010.

CARDOSO, Marcelo de Campos. Usabilidade: aula 7-8. Slideshare. 06 de Julho de 2011.

Disponível em

$<$ https://pt.slideshare.net/marcellocardoso/usabilidad e-78-teste-de-usabilidade> Acessado em 19 de Julho de 2018 .

\section{CARQUEIJA, Andreia Paralta. Ferramentas de} Teste de Usabilidade para Rápidos Feedback Iniciais. 22 de agosto de 2016. Disponível em $<$ https://webdesign.tutsplus.com/pt/articles/usability -testing-tools-for-quick-and-early-feedback--cms-27 049> Acessado em 18 de julho de 2018.

CHYNAŁ, Piotr; SOBECKI, Janusz. 2015. Statistical Verification of Remote Usability Testing Method. Wroclaw University of Science and Technology.

\section{HARTSON Rex H. et. al., Remote Evaluation:} The Network as an Extension of the Usability Laboratory, in CHI96 Conference Proceedings, 1996.

HUNGRIA, Camila. Pegando carona nas grandes marcas. São Paulo. 2014. Disponível em:

$<$ http://www.dcomercio.com.br/categoria/tecnologia /pegando_carona_nas_grandes_marcas/> Acessado em 13 de julho de 2018> Acessado em 22 de julho de 2018.

JORGE, Gabriela Andre e MIYAKE, Dario Ikuo.

\section{Estudo comparativo das ferramentas para} mapeamento das atividades executadas pelos consumidores em processos de serviço. 17 de novembro de 2014. Disponível em $<$ http://www.scielo.br/pdf/prod/2015nahead/0103-6 513-prod-0103-6513128413.pdf $>$ Acessado em 14 de julho de 2018. 


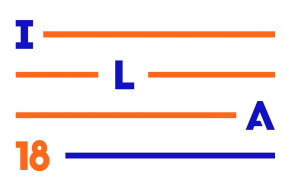

KITCHENHAM, Barbara. 2004. Procedures for Performing Systematic Reviews. Keele University Technical Report TR/SE-0401. Disponível em http://www.inf.ufsc.br/ aldo.vw/kitchenham.pdf

KRUG, Steve. Não me faça pensar: uma abordagem de bom senso à usabilidade na web. 2. ed. Rio de Janeiro: Alta Books, 2008.

LESLIE, Carolina. Panorama UX 2017. Disponível em

$<$ https://www.slideshare.net/lulileslie/panorama-uxwiad2018-89023872> Acessado em 22 de julho de 2018.

LESLIE, Carolina. Teste de usabilidade remoto. 21/01/2016 Disponível em $<$ http://www.saiba-mais.com/2016/01/12/teste-de-us abilidade-remoto/> Acessado em 18 de julho de 2018

MADEIRA, Paulo. Benchmarking : a arte de copiar. Jornal do Técnico de Contas e da Empresa (JTCE). 1999, ISSN 0870-2241. Ano 32, no 411, p. 364-367.

OWEN, Mariana. Metodologias de Projeto e Avaliação do Design de Interação: Aplicação em contexto real por equipas de dimensão reduzida. Dissertação de mestrado, Universidade do Porto, Setembro de 2016. Disponível em $<$ https://sigarra.up.pt/feup/pt/pub_geral.show_file?pi gdoc_id=866831 $>$ Acessado em 18 de julho de 2018.

SCHADE, Amy. Remote Usability Tests: Moderated and Unmoderated. Nilsen Norman Group, 12 de Outubro de 2013. Acessado em $<$ https://www.nngroup.com/articles/remote-usability -tests/> Acessado em 16 de julho de 2018

SEBRAE. Conheça as vantagens do e-marketplace para os pequenos negócios. Disponível em: $<$ http://www.sebrae.com.br/sites/PortalSebrae/artigo s/Conhe $\%$ C3\%A7a-as-vantagens-do- e $\%$ E2\%80\%93marketplace-para-os-pequenos-neg $\%$ C3\%B3cios> Acessado em 15 de julho de 2018.

TEIXEIRA, Fabricio. O que é UX Design? Experiência do usuário explicada em 10 vídeos. UX Collective BR, 17 de julho de 2016. $<$ https://brasil.uxdesign.cc/o-que-é-ux-design-experi ência-do-usuário-explicada-em-10-v\%C3\%ADdeos161be4e5b313> Acessado em 16 de julho de 2018

TEIXEIRA, Fabricio. Triplica a demanda por especialistas em UX no Brasil: mas o que isso significa?. UX Collective BR, 14 de julho de 2017. $<$ https://brasil.uxdesign.cc/triplica-a-demanda-por-e specialistas-em-ux-no-brasil-mas-o-que-isso-signific a-aa31e7904017> Acessado em 16 de julho de 2018

VASSÃO, Caio. (2010) Metadesign: ferramentas, estratégias e ética para a complexidade. São Paulo: Blucher.

VILHA, Anapatrícia Morales. E-marketing para bens de consumo durável. Rio de Janeiro: Editora FGV, 2002 